\title{
ADDENDUM
}

\section{Observation of the inverse Doppler effect in negative-index materials at optical frequencies}

Jiabi Chen, Yan Wang, Baohua Jia, Tao Geng, Xiangping Li, Lie Feng, Wei Qian, Bingming Liang, Xuanxiong Zhang, Min Gu \& Songlin Zhuang

Nature Photon. 5, 239-242 (2011); published online 6 March 2011; corrected after print 3 June 2011.

Since the publication of the above Letter, the authors have noticed a relevant manuscript that was published in October 1997:

Liu, W. F., Russell, P. St. J. \& Dong, L. Acousto-optic superlattice modulator using a fiber Bragg grating. Opt. Lett. 22, 1515-1517 (1997).

The manuscript describes positive and negative Doppler shifts at optical frequencies in an acousto-optic superlattic modulator using a fibre Bragg grating. Associated amendments have been made to the text, a citation to this work has been added and the references have been renumbered accordingly. This revision has been made to the HTML and PDF versions of the Letter. 